\title{
ACL Prehabilitation Improves Postoperative Strength and Motion and Return to Sport in Athletes
}

\author{
Jamie Cunha, D.P.T., and Daniel J. Solomon, M.D.
}

Prehabilitation prior to anterior cruciate ligament reconstruction should include quadriceps strengthening-improving range of motion and balance and proprioception as a minimum. Although the content and duration of prehabilitation varies in the literature, when faced with a delay between diagnosis and surgery, 4-6 weeks of prehabilitation can improve early to mid-term strength and motion and can improve the timing and odds of a patient returning to sport.

\section{Introduction}

A successful anterior cruciate ligament (ACL) reconstruction surgery and rehabilitation optimizes patient satisfaction, knee stability, and return to sport, while also preventing rerupture. Failure occurs in $5-25 \%$ of patients when one considers both graft ruptures and ongoing laxity. The prospect and challenge of going through a revision surgical procedure because of one of these adverse outcomes, with 9-12 months of recovery, obviously must be avoided.

The goals of a physical therapy routine prior to ACL reconstruction, known as prehabilitation, address the most common early deficits after ACL injury: loss of motion, quadricep weakness, and instability. In addition, a prehabilitation program should improve balance, proprioception, and confidence, which helps prevent reinjury or contralateral injury after reconstruction.

California Orthopedics and Spine / EXOS Physical Therapy, Larkspur, California, U.S.A. (J.C.); and California Orthopedics and Spine, Larkspur, California, U.S.A. (D.J.S.).

The authors report the following potential conflicts of interest or sources of funding: D.J.S. is an associate editor of the Journal of Arthroscopy, and he is a consultant for Arthrex, outside the submitted work. Full ICMJE author disclosure forms are available for this article online, as supplementary material.

Received August 31, 2021; accepted November 2, 2021

Address correspondence to D. J. Solomon, M.D., California Orthopedics and Spine, 18 Bon Air Rd., Larkspur, CA, 94939, U.S.A. E-mail: dsolomon@ caorthospine.com

(C) 2021 THE AUTHORS. Published by Elsevier Inc. on behalf of the Arthroscopy Association of North America. This is an open access article under the CC BY-NC-ND license (http://creativecommons.org/licenses/by-nc-nd/4.0/). 2666-061X/211276

https://doi.org/10.1016/j.asmr.2021.11.001

\section{Re-Rupture Rates}

Rerupture rates were evaluated by Samuelsen et al., who performed a meta-analysis, including 47,613 ACL reconstructions $[39,768$ bone-tendon-bone (BTB) and 7,845 hamstring (HS)] from 25 different studies. Mean follow-up was $68 \pm 55$ months.

Overall, $2.80 \%$ BTB grafts ruptured compared with $2.84 \%$ in the hamstring group. Laxity was slightly higher in the BTB group compared to the HS group, but the difference was not significant. ${ }^{1}$

In a systematic review, Grassi et al. reported as many as $8 \%$ of patients undergoing ACL reconstruction will undergo a subsequent revision procedure. Regarding revision ACL reconstruction, when considering the sum of reruptures and objective clinical failures, the proportion of failed revision ACL reconstruction was more than $20 \%$ in 5 of the 15 included series in their review. ${ }^{2}$

Crawford et al. identified 14 studies for review of long-term ACL reconstruction failures. At longer than 10 years clinical follow-up, the reported ACL graft rupture rate was $6.2 \%$, and clinical failure occurred in $\sim 10.3 \%$. At least 1 in 9 patients undergoing ACL reconstruction will have rerupture or clinical failure at long-term follow-up. ${ }^{3}$

\section{Return to Sport Rates}

Return to sport after ACL reconstruction, depending on the study, runs around $90 \%$. This means $10 \%$ of patients never return to the same level of sports after ACL reconstruction surgery and rehabilitation. ${ }^{4}$ Webster et al. found that only $24 \%$ of individuals returned to their preinjury level of sport, despite $91 \%$ reporting preoperatively that they expected to return. ${ }^{5}$ Graincher and Scherr advocate for an assessment of risk factors to determine whether a prehabilitation program prior to ACL reconstruction would benefit the 
patient better than early surgery. Elite and amateur athletes have low return to sport or return to competition rates, despite several studies attempting to develop evidence-based protocols for rehabilitation after ACL reconstruction. The authors cite low preoperative leg symmetry index, weak quadriceps and hamstrings, female gender, older age, fear of reinjury, low self-efficacy, and long waiting times without any intervention between injury and surgery as risk factors for lower return to sport rates. They believe that identifying these risk factors can potentially help with decision making in the modality of prehabilitation and the timing of surgery. They suggest that prehabilitation prior to ACL reconstruction may decrease the loss of functional capacity by restoring neuromuscular control, knee strength, knee and joint position sense, while lowering pain levels and psychologically preparing athletes for return to sport at a similar level to preinjury. Early ACL reconstruction is preferred in elite athletes in order to avoid ingraining of perception of instability and giving-way phenomena, so the authors recommend more analysis of whether prehabilitation would benefit the athlete so much so as to outweigh these risks of waiting. ${ }^{6}$

On the basis of these studies, the scope and scale of recurrent issues after ACL reconstructions are somewhat daunting when you consider the number of ACL surgeries performed annually. Anything we can do to mitigate the risk of reinjury or contralateral injury is helpful. Therefore, our purpose is to review the current literature, including options and success, regarding ACL prehabilitation therapy. There is good support for optimizing the patients' knee flexibility, strength, and proprioception preoperatively and that these prehabilitation physical therapy measures will result in a decrease in ACL reinjury after reconstruction.

\section{Components of Prehabilitation}

What is a good prehabilitation routine? What should be done, when and for how long and how often? What should the goals of prehabilitation be? (Table 1), Should surgery be delayed in lieu of prehabilitation? After reviewing the literature, there does not seem to be much consensus regarding these questions. However, once there is a determination that ACL surgery is necessary, there is almost always at least a brief period before surgery is performed during which prehabilitation can be facilitated.

Shaarani et al. compared outcome measures between a group of subjects that completed 6 weeks of prehabilitation versus control group subjects who did almost nothing prior to ACL surgery. The prehab group performed both strength and balance exercises, with a prescription of 3 sets of 12 repetitions, increasing by 10 $15 \%$ volume-load each week. The authors found that patients who underwent the 6 weeks of prehabilitation scored significantly higher on the single-legged hop test, as well as on their self-reported assessment using the Modified Cincinnati Knee Rating System. These effects were sustained at 12 weeks postoperatively. The mean return to sport timeframe was 34.2 weeks for the prehab group and 42.5 weeks for the control group. ${ }^{7}$

Grindem et al. recommended a standard prehabilitation procedure after isolated ACL rupture for 5 weeks of progressive training, including heavy resistance training, plyometrics, and neuromuscular exercises, aiming at regaining $90 \%$ of quadriceps and hamstring strength and hop performance of the uninjured leg before surgery. ${ }^{8}$

When comparing the Multicenter Orthopedics Outcome Network (MOON) and Delaware-Oslo ACL Cohorts, Failla et al. identified better IKDC and Knee injury and Osteoarthritis Outcome Score (KOOS) scores and higher return to sport rates 2 years after ACL reconstruction (ACLR) in patients undergoing preoperative rehabilitation with neuromuscular training compared to those who did not undergo preoperative rehabilitation. In their assessment, delaying surgery for 10 sessions of prehabilitation could improve functional knee scores by $12 \%$ to $15 \%$. Overall, prehabilitation seems to be a positive alternative to passive waiting periods between injury and reconstruction.?

\section{Outcomes of Prehabilitation}

A good indicator for potential successful prehabilitation is the ability to achieve $80 \%$ quadricep strength compared to the unaffected side. Patients who achieve this milestone minimize their chance of persistent strength differences for up to 2 years postsurgery. ${ }^{10}$

A systematic review by Giesche et al. evaluated the benefits of prehabilitation on objective, self-reported and return-to-sport-specific outcomes, in comparison to ACL postoperative rehabilitation without prehabilitation. They found low-level evidence that supports the notion that prehabilitation improves returnto-sports rates and 2-year postoperative knee function.

The time before surgery ranged from 5 to 9 months, and prehabilitation ranged from 4 to 6 weeks (4.8 weeks average), 2-4 times per week, for a maximum of 75 to 120 minutes. The prehabilitation included lower limb open- and closed-chain exercises, neuromuscular training (perturbation, balance, stability, proprioceptive exercises), quadriceps-focused muscle control, and cocontraction exercises, as well as stretching and range of motion (ROM) exercises for the lower limb. Some studies included as part of their prehabilitation regimen: patellar mobilization, kinesiotaping of the patellofemoral joint and plyometrics (single leg hops with soft landing).

In the various studies, prehabilitation improved maximal quadriceps torque, single-leg-hop for distance, 
Table 1. Sample ACL Prehabilitation Program Beginning Six Weeks Before Reconstruction

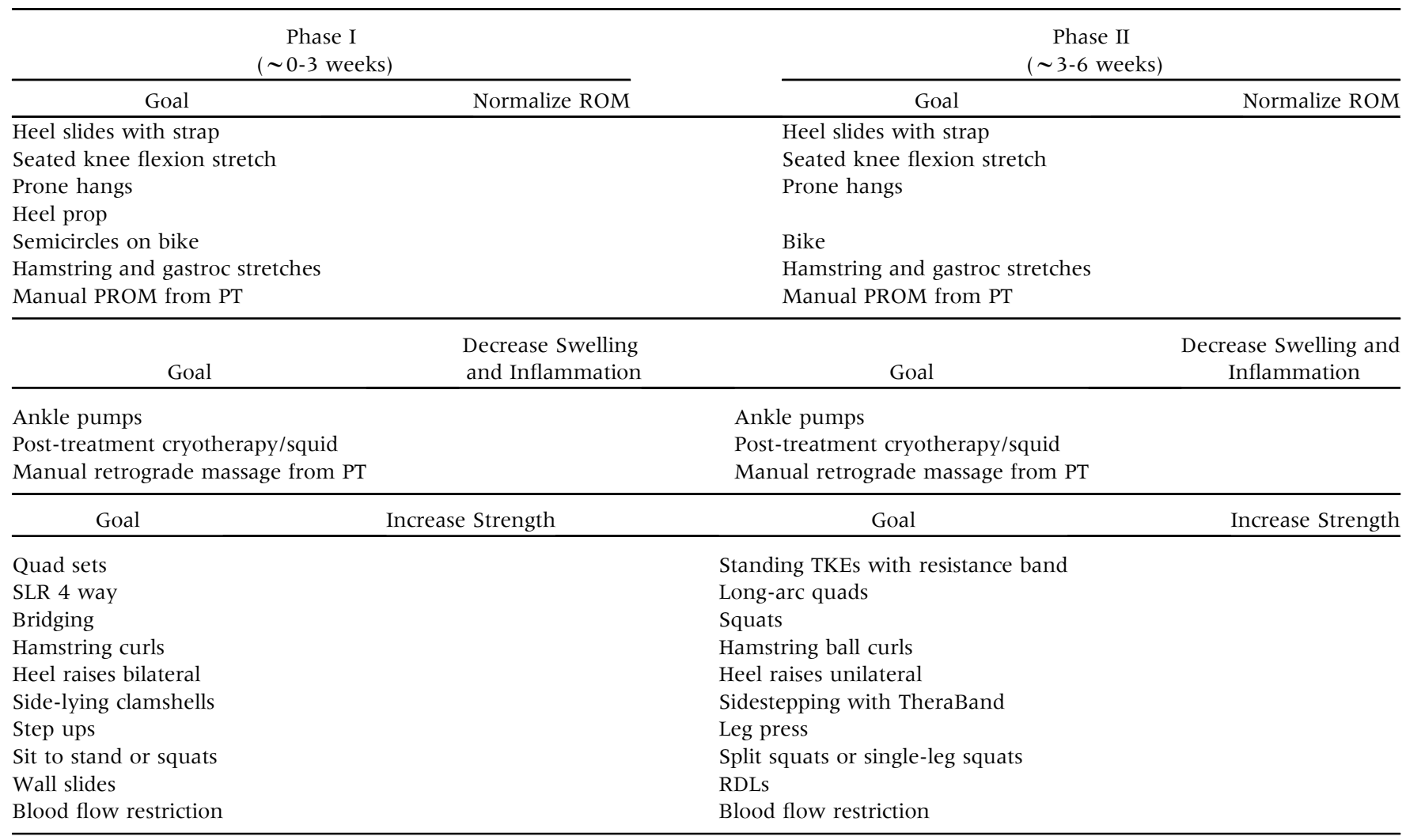

\begin{tabular}{clll}
\hline Goal & Improve Balance/Proprioception & Goal & Improve Balance/Proprioception \\
\hline
\end{tabular}

Single-leg balance

Tandem stance

Single-leg balance on uneven surfaces

Double-leg balance on uneven

Tandem walk

surface

Single-leg balance star taps

Also identify any proximal and distal joint restrictions in range of motion (ROM) and strength, such as hip extension/external rotation, hip flexion/internal rotation, ankle dorsiflexion, and thoracolumbar spine ROM).

If you have a dynamometer, measure unaffected limb open-chain knee extension strength for post-op limb symmetry index.

and limb symmetry index. There was also improved self-reported knee function, return to sport tended to be faster, and the prehabilitation groups had a higher return-to-sport rate at 2 years postsurgery. ${ }^{11}$

\section{Discussion}

While prehabilitation seems to be beneficial overall, the results may not endure. Reddy et al. compared the results of 41 patients who were randomly assigned to prehabilitation versus standard rehabilitation protocols in postoperative ACL reconstruction (quadruple hamstring graft). Twenty patients had prehabilitation with goals to decrease pain, swelling and inflammation; to restore range of motion; and to restore muscle strength. The secondary goals of prehabilitation were eliminating antalgic gait and improving neuromuscular control. The prehabilitation regimen included quadriceps-strengthening exercises, mini-squats, straight leg raising, hamstring stretches, ankle pumps, and ice application after exercises. The authors stated that a 4-week program significantly improved knee extensor muscle strength postoperatively and improved knee function by assessing Single Leg Hop Test distance. The prehabilitation group experienced a significantly improved range of motion at 3 and 6 weeks versus the standard rehabilitation group, but there was no difference at 3 and 6 months. There was no statistical difference in Lysholm or IKDC scores between the groups. ${ }^{12}$

Other studies suggest that prehabilitation is more successful, and benefits may last substantially longer than those found by Reddy et al. Patients who complete prehabilitation may manifest improvements for up to 6 years postoperatively compared to those that do not do a preoperative exercise routine. These improvements apply across knee-related strength, range of motion, function, and outcome measure scores, including Lysholm scores, Tegner activity scores, the lower extremity functional scale, and the functional hop test (Table 2). ${ }^{13,14}$ 
Table 2. Advantages of ACL Prehabilitation

Goals and Benefits of ACL Prehabilitation

1. Restore knee range of motion

2. Restore lower extremity strength, especially quadriceps

3. Restore knee stability

4. Restore balance

5. Restore proprioception

6. Restore confidence in return to sport

7. Reduce likelihood of reinjury or contralateral injury

Yalfani et al. performed a systematic review of 10 studies to examine whether preoperative exercises can affect self-reported knee function outcomes. The authors found that several preoperative programs significantly enhanced self-reported knee function after surgery. They concluded that preoperative rehabilitation, consisting of progressive strengthening and neuromuscular training, followed by a postoperative rehabilitation program, had greater functional outcomes after ACLR and that preoperative rehabilitation should be considered as an addition to the standard of care to maximize functional outcomes after ACLR. The authors discussed that even if ACLR creates good stability, the quadriceps strength defect is reported as one of the limiting elements in returning to preinjury phase, which can last for up to 2 years after surgery.

Preoperative training in this systematic review varied in duration, frequency, and content; mean training was 4 weeks duration (average frequency of 3 times per week), and the content included quadriceps and hamstring strength training; proprioception; and neuromuscular, balance, and gait training. Most of the studies evaluated knee function through questionnaires. Studies reviewed showed that muscle function maximization and ROM deficit minimization preoperatively translate into improved postoperative outcomes. They predicted that with better strength and performance, prehabilitation can prevent reinjury.

They concluded that there was moderate-quality evidence that prehabilitation exercises, based on neuromuscular exercises with strength training could enhance self-reported function and that maximizing quadriceps strength with progressive preoperative exercises should be the focus for therapists, in order to enhance functional outcomes after ACL reconstruction. ${ }^{15}$

\section{Future Directions}

While most studies evaluate the effectiveness of prehabilitation on physical outcomes, Carter et al. also looked at psychological outcomes following ACL reconstruction through a systematic review. The authors suggested that return to sport after ACL reconstruction is commonly inadequate because patients aren't mentally ready despite having achieved a successful functional outcome. Prehabilitation programs don't commonly address the mental aspect related to ACL rehabilitation. ${ }^{16}$

Visualization techniques and virtual reality training could be used to boost confidence and mental preparedness for return to sport.

\section{Conclusion}

Although no study seems to provide a consensus on what prehabilitation encompasses, the basic components should include improvement in knee ROM, quadricep strengthening, and proprioception. The duration can be as short as 3 weeks, but the more successful programs last at least 4-6 weeks. In some patients, it may be beneficial to delay surgery to optimize these knee functions. Benefits of a prehabilitation program (better ROM and quadriceps strength) last at least 6 weeks, but perhaps as long as 6 years with the hope that a vigorous prehabilitation program prevents reinjury or contralateral injury after ACL reconstruction.

\section{References}

1. Samuelsen BT, Webster KE, Johnson NR, et al. Hamstring autograft versus patellar tendon autograft for ACL reconstruction: Is there a difference in graft failure rate? A meta-analysis of 47,613 patients. Clin Orthop Relat Res 2017;475:2459-2468.

2. Grassi A, Kim C, Marcheggiani Muccioli GM, et al. What is the mid-term failure rate of revision ACL reconstruction? A systematic review. Clin Orthop Relat Res 2017;475: 2484-2499.

3. Crawford SN, Waterman BR, Lubowitz JH. Long-term failure of anterior cruciate ligament reconstruction. Arthroscopy 2013;29:1566-1571.

4. Harris JD, Abrams GD, Bach BR, et al. Return to sport after ACL reconstruction. Orthopedics 2014;37:e103-el08.

5. Webster KE, Feller JA. Expectations for return to preinjury sport before and after anterior cruciate ligament reconstruction. Am J Sports Med 2019;47:578-583.

6. Granicher P, Scherr J. Do athletes benefit from preoperative physical therapy before ACL-reconstruction? Sports Orthopaed Traumatol 2021;37:126-131.

7. Shaarani SR, O'Hare C, Quinn A, Moyna N, Moran R, O'Byrne JM. Effect of prehabilitation on the outcome of anterior cruciate ligament reconstruction. Am J Sports Med 2013;41:2117-2127.

8. Grindem H, Granen LP, Risberg MA, et al. How does combined preoperative and postoperative rehabilitation programme influence the outcome of ACL reconstruction two years after surgery? A comparison between patients in the Delaware-Oslo ACL Cohort and the Norwegian National Knee Ligament Registry. Brit J Sports Med 2015;49:385-389.

9. Failla MJ, Logerstedt DS, Grindem H, et al. Does extended preoperative rehabilitation influence outcomes 2 years 
after ACL reconstruction? A comparative effectiveness study between the MOON and Delaware-Oslo ACL COhorts. Am J Sports Med 2016;44:2608-2614.

10. Eitzen I, Holm I, Risberg M. Preoperative quadriceps strength is a significant predictor of knee function two years after anterior cruciate ligament reconstruction. Brit J Sports Med 2009;43:371-376.

11. Giesche F, Niederer D, Banzer W, Vogt L. Evidence for the effects of prehabilitation before ACLreconstruction- on return to sport-related and selfreported knee function: A systematic review. PLoS ONE 2020;15:e0240192.

12. Reddy DVS, Kamath SU, Annappa R, Krishnamurthy SL, Kamath K, Mallya S. Does preoperative rehabilitation give better short-term results in anterior cruciate ligament reconstruction? Amb Surg 2020;26.2:40-43.
13. Amaravati R, Sekaran P. Does preoperative exercise influence the outcome of ACL reconstruction? Arthroscopy 2013;29:el82-el83.

14. Alshewaier S, Yeowell G, Fatoye F. The effectiveness of preoperative exercise physiotherapy rehabilitation on the outcomes of treatment following anterior cruciate ligament injury: A systematic review. Clin Rehab 2016;31:34-44.

15. Yalfani A, Ebrahimi Atri A, Taghizadeh Kerman M. The effect of prehabilitation on the self-reported outcomes of anterior cruciate ligament reconstruction: A systematic review. Iran J Health Sci 2021;9:45-55.

16. Carter HM, Littlewood C, Webster KE, Smith BE. The effectiveness of preoperative rehabilitation programmes on postoperative outcomes following anterior cruciate ligament reconstruction: A systematic review. BMC Musculoskelet Disord 2020;21:647. 